\title{
Pulse Shaping-Assisted Nonlinear Spectral Broadening
}

\author{
Xin Yang ${ }^{1}$, David J. Richardson ${ }^{1}$ and Periklis Petropoulos ${ }^{1}$ \\ 1. Optoelectronics Research Centre, University of Southampton, Southampton, United Kingdom, SO17 1BJ
}

Recent developments in optical fibre technology have allowed significant advances in the nonlinear generation and tailoring of broadband spectra. Much work in this direction has focused on the optimisation of the properties of the optical fibres used for the nonlinear pulse propagation. In this work, we combine the nonlinear element (a highly nonlinear fibre - HNLF) with a programmable phase and amplitude filter, which we use as a pulse shaper. This architecture allows power-efficient and flexible sculpturing of the output spectrum, which is no longer tied directly to the characteristics of the pulse source used at the input. The pulse shaping function is obtained by application of the inverse split-step Fourier method (ISSFM) [1], which uses the desired output spectrum and the HNLF characteristics as its input parameters [2]. As an example of the application of this technique, we study the generation of broadband flat (third-order super-Gaussian) spectra starting from a $10 \mathrm{GHz}$ mode-locked pulsed laser (MLL). Here we present our design procedure and a first experimental validation of the technique.

Fig. 1 schematically illustrates the methodology we followed. In the simulation, we started with the time domain expression corresponding to the target spectrum as shown in Fig. 1(c). The pulse width $T_{0}$ and chirp parameter $C$ formed the control parameter set for the simulation and led to output spectra of various bandwidths. By applying the HNLF parameters (see Fig. 1) into the ISSFM, we were able to calculate the pulse characteristics yielding these spectra (see Fig. 1(b)). The results were related to the MLL pulse characteristics (Fig. 1(a)) by calculating the ratios of the root mean square (RMS) of both the pulse widths $R_{\tau}^{\text {shaped }}\left(T_{0}, C\right)$ and the spectral widths $R_{\lambda}^{\text {shaped }}\left(T_{0}, C\right)$ between the shaped and MLL pulses. The quantities were drawn on contour plots, mapped against parameters $T_{0}$ and $C$, as shown in Fig. 2(a) and (b). In practice, the constraints $R_{\tau}^{\text {shaped }}\left(T_{0}, C\right)<1 /$ dutycycle (dutycycle $=0.1$ in our experiment) and $R_{\lambda}^{\text {shaped }}\left(T_{0}, C\right)<1$ are applied to ensure that the width of the shaped pulses is less than the MLL pulse period and that they can be carved within the pulse bandwidth.

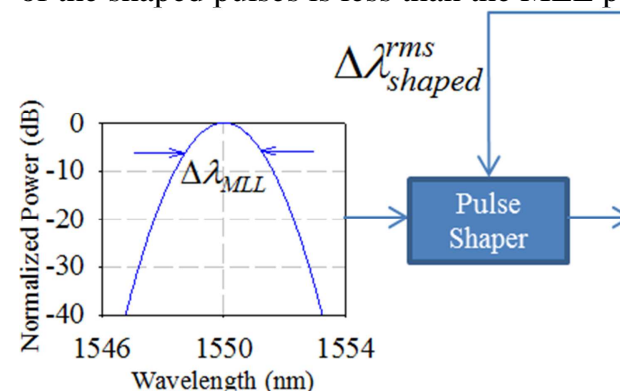

(a)

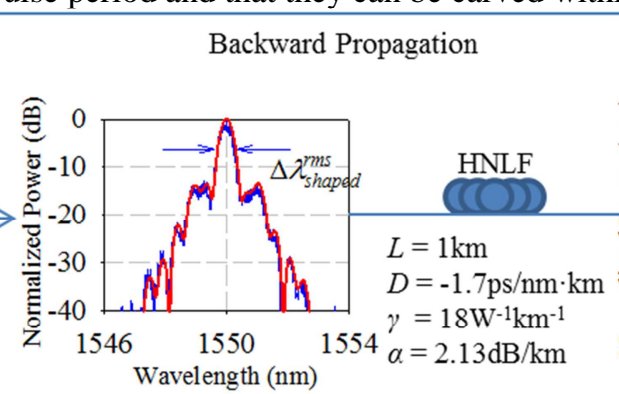

(b)

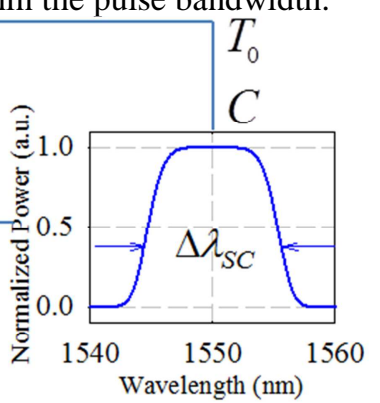

(c)

Fig. 1 A schematic of the simulation and experimental procedure. (a) Spectrum of the MLL source. (b) Spectra of the pulses after shaping. The red line represents the simulation curve, while the blue line is the experimental result after shaping. (c) Target spectra with third-order super-Gaussian shapes.

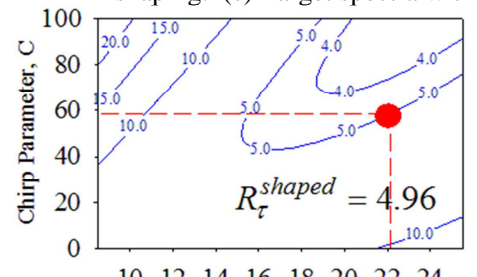

$\begin{array}{llllllll}10 & 12 & 14 & 16 & 18 & 20 & 22 & 24\end{array}$ Pulse Width of the Waveform of Target SC, $\mathrm{T}_{0}(\mathrm{ps})$

(a)

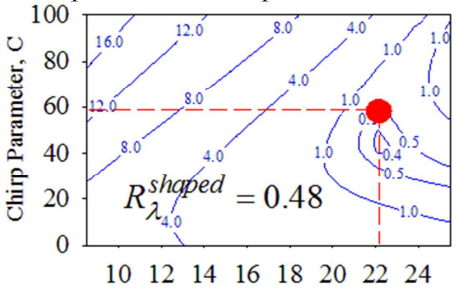

Pulse Width of the Waveform of Target SC, $\mathrm{T}_{0}$ (ps)

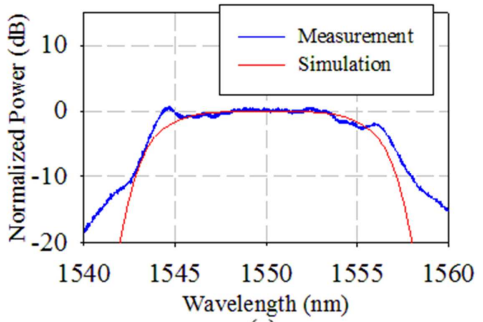

(c)

Fig. 2 Simulation and experimental results for pulses with a peak power of $2 \mathrm{~W}$. (a) Contour plot of $R_{\tau}^{\text {shaped }}\left(T_{0}, C\right)$. (b) Contour plot of $R_{\lambda}^{\text {shaped }}\left(\mathrm{T}_{0}, C\right)$. The red dots in (a) and (b) represent the position of the chosen control parameter set in the parameter space. (c) The simulation and measured results of the spectra with third-order super-Gaussian shapes.

The contour maps allow us to choose those shaped waveforms that satisfy the two constraints. In our experimental demonstration, we have chosen the condition represented by the marked values in Fig. 2, which correspond to $T_{0}=22.1 \mathrm{ps}$ and $C=59$ and yield a $12 \mathrm{~nm}$ super-Gaussian spectrum. The corresponding shaping function was fed to the programmable pulse shaper (Finisar Waveshaper) and the generated spectrum was measured at the output of the HNLF. The measured spectra are compared with the simulation in Fig. 2(c). Note that the programmable nature of the pulse shaper should allow measurements of the difference between the desired and obtained spectra to be used to provide adaptive feedback into the shaping procedure in order to compensate for potential sources of error e.g. uncertainty in the MLL pulse or fibre characteristics.

\section{References}

[1] Shuxian, et al., IEEE LEOS Annual Meeting Conference Proceedings, p. 10.1109/LEOS.1999.811876, 1999.

[2] Almeida, P.J., et al., Optical Fiber Communications Conference Technical Digest, p. 3 pp. Vol. 4|6 vol. (2200), 2005. 